\title{
An Overview of the Development of Novel Textile Design by the Application of Creative Components of the Chakma Community of the Chittagong Hill Tracts in Bangladesh
}

\author{
Chakma Arpan, Xinbo Ding, Shuying Jiang, A. F. M. Fahad Halim, Hossen Md. Muzammel \\ Department of Textile Science \& Engineering, College of Materials and Textiles, Zhejiang Sci-Tech University, \\ Hangzhou, China \\ Email: arpanzstu17@yahoo.com
}

How to cite this paper: Arpan, C., Ding, X.B., Jiang, S.Y., Halim, A.F.M.F. and $\mathrm{Mu}-$ zammel, H.Md. (2020) An Overview of the Development of Novel Textile Design by the Application of Creative Components of the Chakma Community of the Chittagong Hill Tracts in Bangladesh. Journal of Textile Science and Technology, 6, 1-10. https://doi.org/10.4236/jtst.2020.61001

Received: November 1, 2019

Accepted: November 24, 2019

Published: November 27, 2019

Copyright $\odot 2020$ by author(s) and Scientific Research Publishing Inc. This work is licensed under the Creative Commons Attribution International License (CC BY 4.0).

http://creativecommons.org/licenses/by/4.0/

\begin{abstract}
In the world, there are more than 370 million indigenous and tribal people with especial culture and traditions. The Chakma's is one of the groups of indigenous people dwelling Chittagong hill tracts in Bangladesh. Chakma's has its own culture, tradition, literature, and costume designs. The design costumes of the Chakma's play an essential role. The Chakma's preserved their textile design in "Alam". Alam is a kind of cloth embroidered with various designs carrying the heritage of the Chakma. The use of Alam began approximately 500 years ago. All used clothing in Chakma society is weaved manually in the back-strap loom, which is generally very time-consumingthis limits its design aesthetics and universal use. Because of globalisation, the use of the traditional design of Chakma's are threatened today. Till today in our country and overseas, no attempts were taken for research of the traditional Chakma's design. The purpose of this research is to upgrade and digitise Chakma's designs. Furthermore, efforts were made for the automatization of the traditional process to preserve traditional Chakma's designs. In this study analysis of the fabric designs, pattern structure, colour applications of the traditional Aalam design were constructed using small prototype Rapier loom technology. We found that most of the single designs of the Alam were possible to weave in small prototype modern loom. On the other hand, intricate designs were not possible to weave in small prototype loom but are possible in the jacquard loom. The key idea is to establish an approach to modernise the traditional weaving process in order to make it convenient, economical and less time-consuming.
\end{abstract}




\section{Keywords}

Aalam, Indigenous Clothes, Traditional Loom, Plain Weave Structure, Small Prototype Machine, Automatic Loom

\section{Introduction}

Bangladesh is a sovereign country located in South Asia. Bangladesh is an up growing star in the textile sector. In recent years, Bangladesh textile products have been highly competitive in the international textile market [1]. According to statistics from relevant government departments of Bangladesh, there are more than 4000 textile and garment processing companies in Bangladesh, and more than 4.8 million skilled and unskilled employees are directly involved with this sector [2]. There are 45 indigenous groups of people who lived in Bangladesh. The Chakma's is the majority of indigenous people who are dwelling in three hilly districts of Chittagong hill tracts in Bangladesh. Chittagong hill tracts are one of the most tourist attractions place in Bangladesh. Life of the Chakma's in Chittagong hill tracts is particularly impressive [3]. Generally, the Chakma's culture is very exclusive for its ethnicity. The Chakma's is different and distinct from the rest of other populations of Chittagong hill tracts and the mainland of Bangladesh in respect of culture, customs, ethnicity, food, language, religion, tradition, and mainly their unique designing clothes [4]. The clothing of Chakma's is the most excellent image of its own culture. Clothing is most famous for the human body, especially protecting, maintaining the temperature, and incorporate the mark of cultural inheritance and the change of fashion aesthetics. Till today no one has shown attention to The Chakma's design for practical research purpose in textile.

On the other hand, the Chakma's designs are unique for their jazzy and mysterious beauty. The Chakma's traditional fabrics are simple, colourful with a good pattern and comfortable fabrics [5]. Most of the Chakma's decorative fabrics are made by cotton, wool, and rayon. In Bangladesh, the Chakma's indigenous culture is excellently influential with regional characteristic, historical development regularity, deep ethic characteristic, etc. [6]. Most of the Chakma's fabrics patterns taken from nature, and integrated into the nation's own emotional and cultural circumstances, but also reflect the natural environment in which the nation, such as traditional Chakma's fabrics often appears in the image of a variety of flower, animals and vegetation, people's gratitude and yearning for the gratitude of natural gifts and useful life. The pattern mostly made of bright colours, but also contains some exciting patterns at the same time related to decorative purpose [7]. Figure 1 shows the traditional Chakma's design fabric.

The traditional culture of the Chakma's is an essential part of a colourful universe. Diverse culture is an invaluable cultural treasure and national pride in all countries. The traditional designing fabrics, costumes are in centric position. It 
Can gleams ideology, political status, social, religious status, economic development, and cultural status of a nation [8]. Chakma's traditional design patterns have deep cultural appellation, and the craftsmanship is exquisite [9]. The Chakma lucrative fabric design style and visualise continuously influenced and significantly increased demand in modern fabric market. But the development of unique Chakma's fabric subject to the scarcity of technical equipment [10]. The production of these fabrics is still done in back-strap loom shown in Figure 2. For this reason, more time required for weaving operations, which results in less production. Very few researchers discussed Chakma's traditional design. However, still there is no practical research carried out for modernization of the traditional weaving process of Chakma's. There are some lackings on how to weave the traditional design in the modern loom by automation, increase the weaving functional efficiency in their research did not come up.

Chakma's textile designs are produced on hand weaving loom and these slow down the production process with limited quality. In this study, endeavours were taken to develop the traditional Chakma's design on the rapier loom in order to modernise the traditional weaving process to reduce weaving time and enhance the quality of the product.

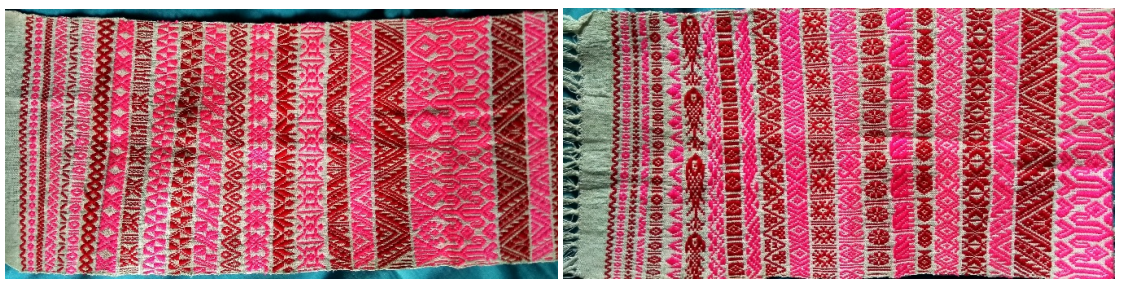

Figure 1. Chakma traditional designs fabric "Alam".

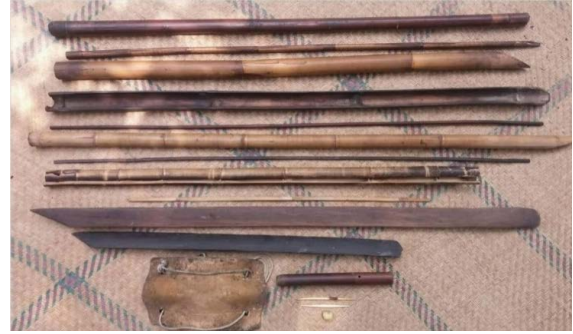

(a)

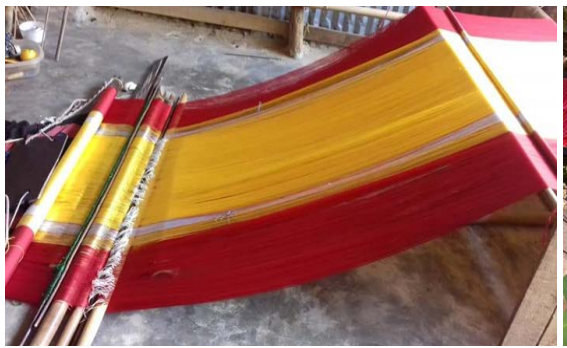

(c)

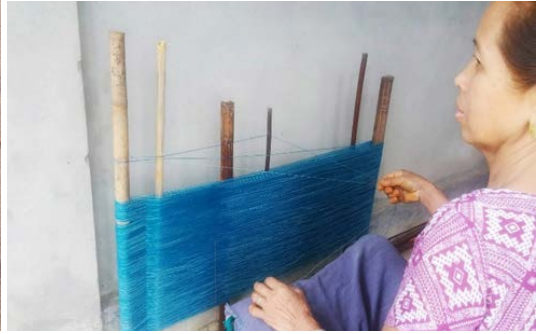

(b)

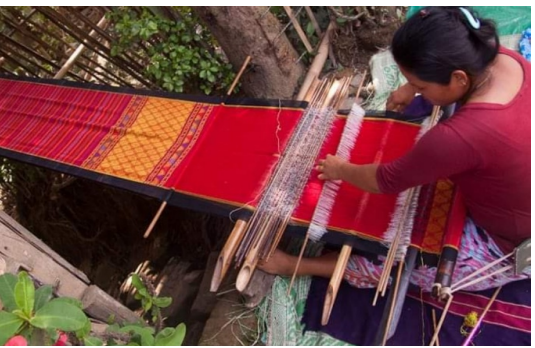

(d)

Figure 2. (a) Tools used in traditional loom; (b) Traditional Loom Preparing; (c) Prepared Loom; and (d) Traditional Back-Strap Loom. 


\section{Materials and Methods}

\subsection{Materials}

Yarns made of cotton, silk, wool, and rayon are mainly used for Chakma's fabrics. Weaving yarns from jute or flax is also used for traditional fabric production. Locally, these yarns are prepared by hand-spun method. Synthetic yarns are also introduced to increase the volume of current production. However, for this study, wool and Cotton yarn was used as raw material.

\subsection{Pattern Selection for Weaving on Fabric}

The traditional Chakma's fabrics have a unique style, and their pattern features, colour combinations have strong ethnic and regional characteristics. Therefore, in the design process, careful selection of the pattern and colour matching of the clothing is required [1]. The origins of these arts are sourced from natural elements such as landscape views, animal patterns, and other natural phenomena from their areas for fabric decoration. In this study, an ancient pattern named "Rajaro Phool", which means "King's Flower" was weaved on fabric shown in Figure 3. This pattern represents the royal family and symbolises noble dignity. The patterns of flowers generally focus on the appearance of the main body of flowers, which is different from other flower patterns. The surrounding also accompanied by geometric figures. The whole pattern guarantees the integrity of the composition while abstracting the flower, and highlights the overall atmospheric beauty. The colour-matched with the solemn red and white, which can better show the grandeur and majesty of the place.

\subsection{Design Elements and Its Combination Principle}

The design creation is a personal expression designed based on individual preferences from elements selection, colour and its aesthetics combining styles to achieve the targeted requirements such as expression of Chakma's own tradition and customs, customer attraction during the selling of products. This part mainly includes the design of the pattern by digitizing through Java Computer-aided design (JCAD) oriented designing and manufacturing system, which will enhance the aesthetic, feel and look of the fabric. According to the number of available heald frames, the design method adopted and digitised on computer pattern software.

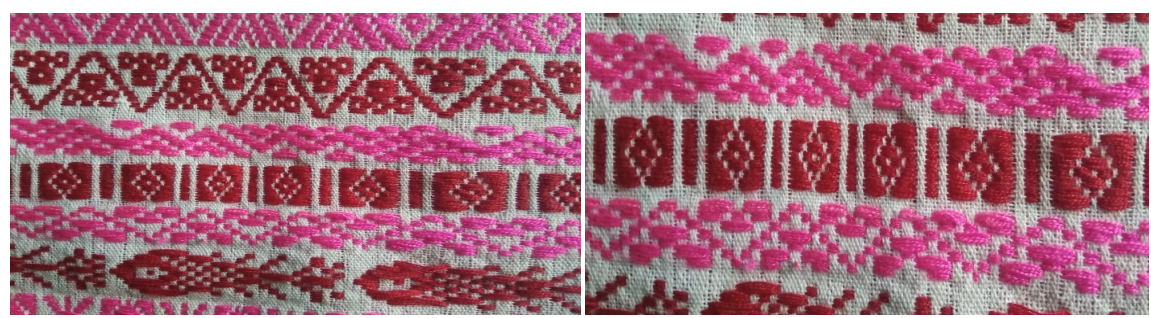

Figure 3. Rajaro phool (King's Flower). 


\subsection{Design Parameter}

The weave structure of the fabric consists of double weft and plain weave. The warp yarn count of the fabric is 46.82 Tex which is white colour cotton yarn, and the red coloured weft yarn count is $33 \mathrm{Nm}$. Three-ply wool yarns used as coloured weft yarns. Since the width of the small prototype machine is fixed and cannot exceed $24.5 \mathrm{~cm}$, the width of the finished product is designed to meet the requirements of weaving conditions, and the inner and outer frames are designed to be $20 \mathrm{~cm}$. In the double weft structure, the red coloured wool thread used as the first colour weft, and the white cotton thread used as the second weft. The weft density of the second weft structure part set as the first weft density, and the plain weave structure part set as the second weft density. The warp density $\mathrm{Kj}$ is $180 / 10 \mathrm{~cm}$, and the first weft density $\mathrm{Kw} 1$ of the weft part is $240 / 10 \mathrm{~cm}$, and the second weft density Kw2 is $120 / 10 \mathrm{~cm}$. Table 1 represents the processing specification of Kings flower.

\subsection{Trial Weaving and Finishing}

In our study, small prototype rapier loom was used for trial weaving process, shown in Figure 4. Most of the current prototypes are semi-automatic single-action multi-arm faucets, 16-piece heald frame, and single warp beams [2]. Three main types of domestic prototypes, namely mechanical prototypes, fully automatic prototypes, and electronic prototypes are available [11]. In this study, we used SGA598 automatic rapier weaving machine, as shown in Figure 5. The prototype uses pneumatic pendulum weft insertion, which can be fully automated, with high production efficiency and well-finished product quality. The equipment can adapt to the sample weaving and product development of various kinds of fabrics. The electro-pneumatic control color selection device has a weft

Table 1. Processing specification of kings flower.

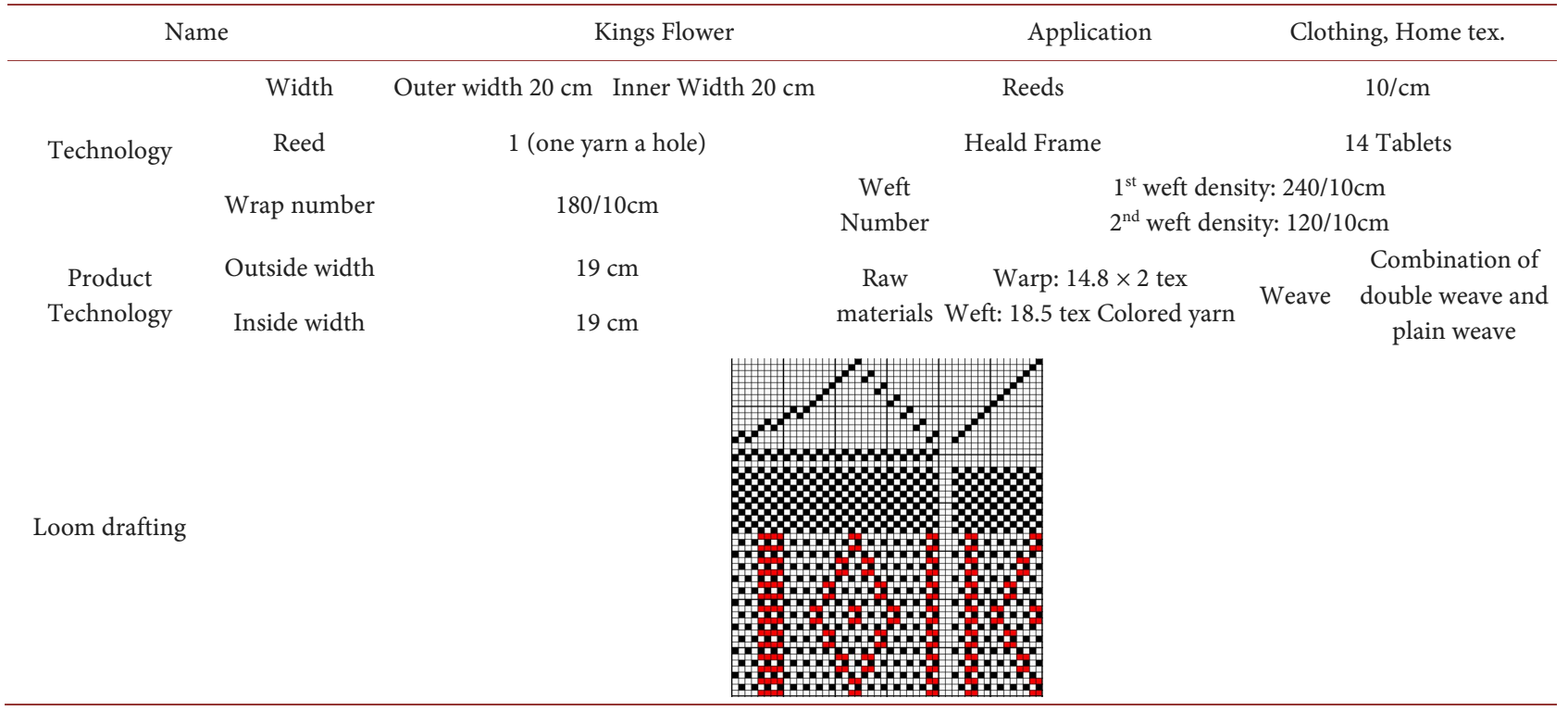




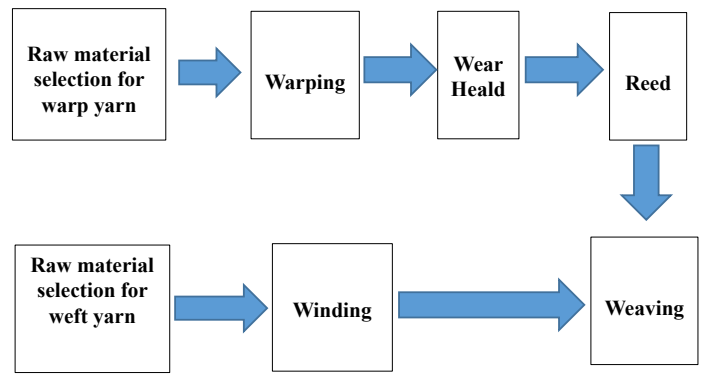

Figure 4. Weaving process automatic rapier machine.

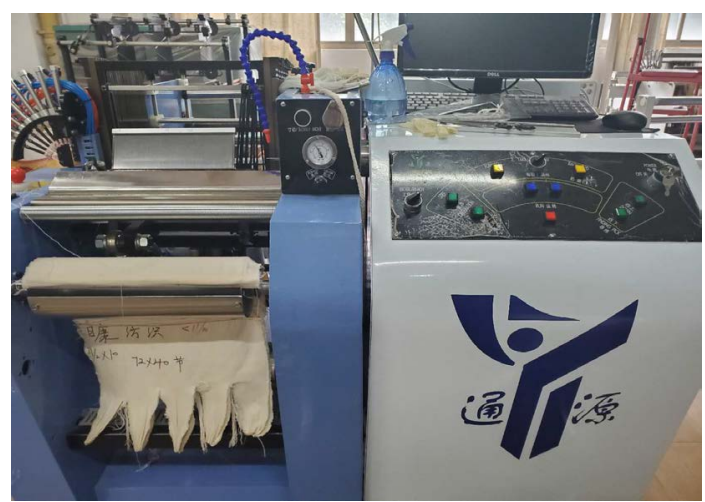

Figure 5. SGA598 Automatic rapier loom.

selection of up to 8 colors and up to four weft densities in the same fabric. The positive electronic delivery method ensures that the tension of the tangle is uniform during the weaving process, pneumatic opening, and the available heddles are 4 to 20 pieces [2].

After completing the raw material preparation work, we choose to wear the heald frame, because of the design series of this study the number of heald frames is large. After the completion of the reed piercing, the process of wearing the reed is carried out. The series of reed piercing designed by single-reed piercing (Figure 6(a)). At the same time, it can be the correctness of the splicing in time according to the graph of reed piercing. If an error occurs, it can correct in time. Finally, the input of the pattern and the setting of each parameter are needed for the control system of the machine, as shown in Figure 6(b). Before weaving, after resetting all parts of the weaving machine, it is necessary to check whether the opening is clear. If it is not, the tension of the warp yarn needs to be continuously adjusted to ensure the clearness of the opening and the smooth running of the weaving. The tension of the yarn is a process parameter involved in each step of the weaving process. Moreover, the tension of the yarn is essential weaving process, which directly affects whether the weaving can proceed smoothly. The yarn tension should keep as constant as possible during the weaving of the fabric [12]. If the tension not controlled correctly, quality problems quickly led to wire breakage and low-speed production [13]. Only the tension can be made within a specific range so that the weaving process can carried out smoothly. The warping process is according to a certain amount, density, 


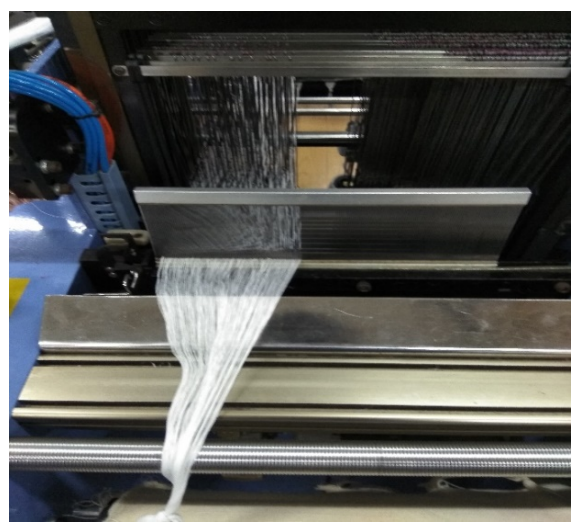

(a)

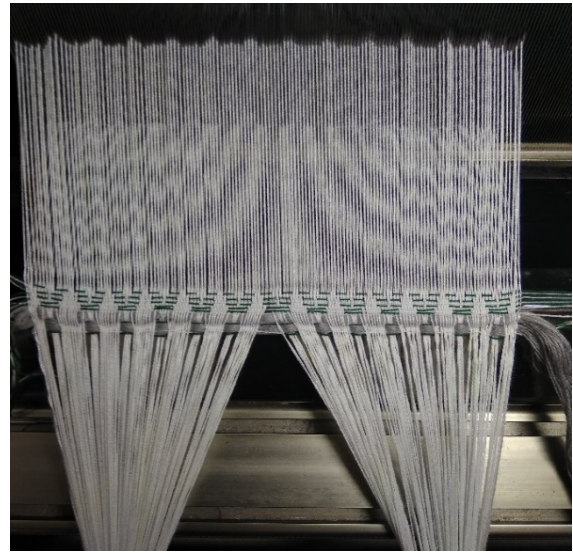

(c)

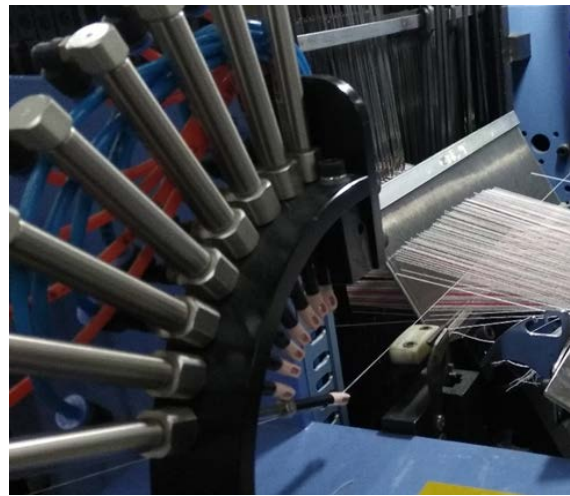

(e)

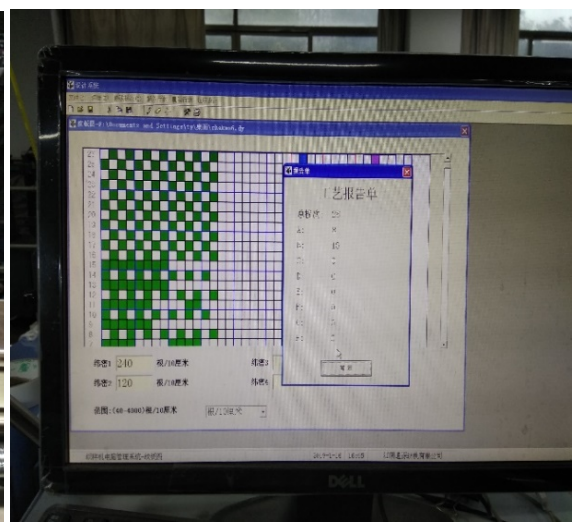

(b)

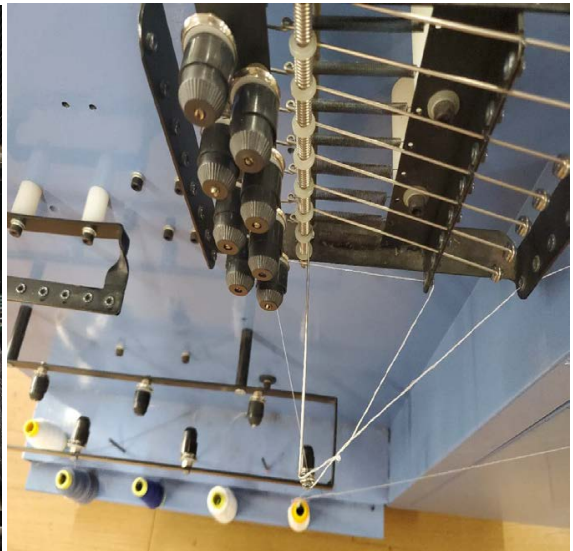

(d)

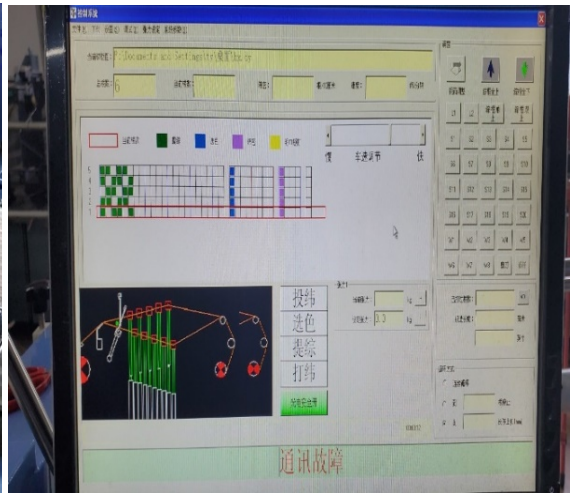

(f)

Figure 6. (a) Yarn piercing heald frame and reed; (b) Pattern organisation; (c) Warp yarn tension; (d) Weft yarn tension; (e) Weft insertion device; and (f) Beating up Display.

width, and technical requirements winding warp yarns into a certain length of warp beam [14]. The key to uniform warping tension is improve warping quality and to ensure uniform warping tension, and it is necessary to ensure that the tension of the single yarn is moderate and the tension of the yarn is uniform [15]. As shown in the Figure 6(c). During the weaving process, the weft yarn tension is also particularly important. Improving the ability to control the weft tension can improve fabric quality and weaving efficiency, while also reducing 
weaving problems [16]. Figure 6(d) The electronic prototype machine operation is similar to that of a mechanical prototype, but it needs to input or select the pattern in the computer before use [11] and save the pattern. Our sample fabrics organization is a plain weave. We used two sets of weft yarns, white and red, in our project. These two sets of weft yarn were placed on the weft insertion device in sequence. Figure 6(e) Then the set weft insertion order of the pattern chart. After setting the pattern, the pattern import into the computer control system. When the machine starts to work, it should select the working state to weave the imported pattern. At the same time, the computer system will display which weft yarn is to hit. Figure 6(f) Before weaving, it should be adjusted all of the equipment. Otherwise, it causes problems for the machine. The opening of the sample loom is controlled by the pattern to control the lifting of the heald frame [17]. The trial weaving work can carry out after all the preparations had completed. If there is no problem for single and circular beating then continues beating can be performed, and all kinds of the action LCD screen will feedback.

When the trial weaving of the traditional design completed, we can develop weaving the sample application of the test by photoshop software. The design can be used in different fields to develop all practical applications. Adjusting the fabric with software, the effect simulation can apply to the desired scene to visualize the application. Figure 7 represents the design after being completed.

In our project, we weaved several types of design patterns from the traditional Chakma's fabric. The traditional Chakma's fabric designs part is the main key part of the total project. In the process of application, we need to grasp the essence of the pattern and redesign the pattern with the existing weaving relatives to weave the unique style of Chakma's clothing fabric as smooth as possible. According to the sample fabric in this project, we used the same properties yarn for all selected patterns and methodology of weaving in a small prototype system, also same for all patterns. The designs displayed below (Figures $8(\mathrm{a})-(\mathrm{c}))$.

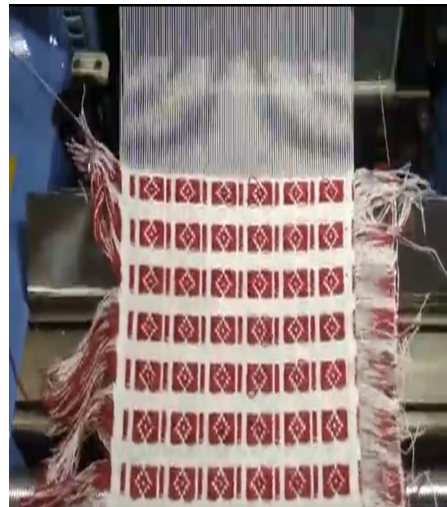

(a)

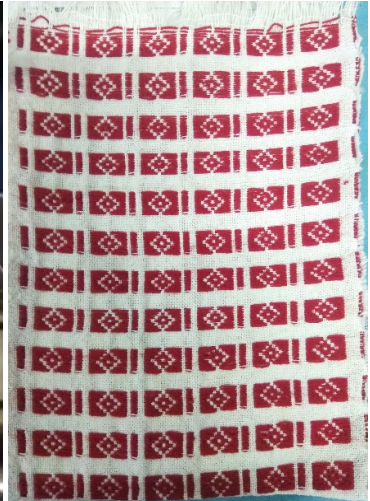

(b)

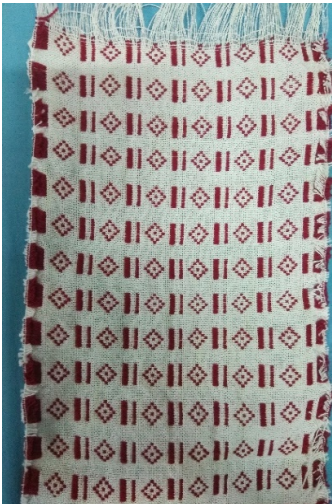

(c)

Figure 7. (a) Complete Design (Kings Flower); (b) Face side; and (c) Backside. 


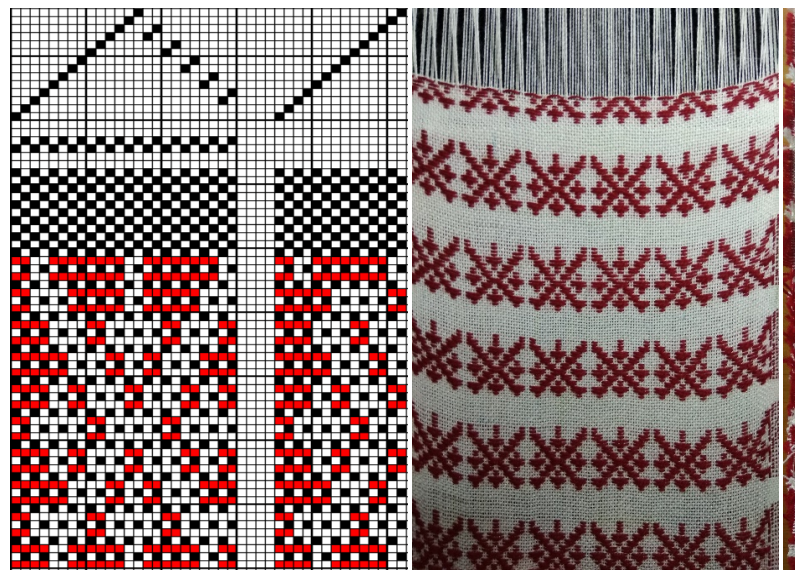

(a)

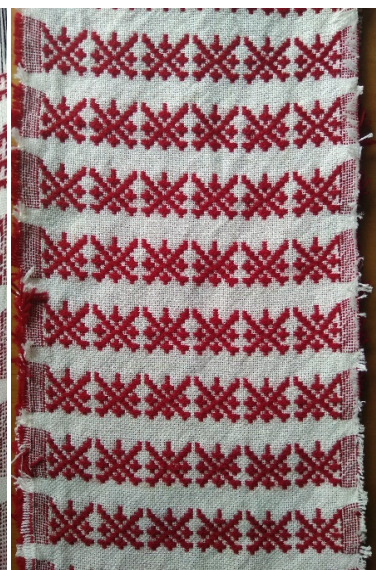

(c)

Figure 8. (a) Drafting \& Lifting; (b) Weaving in Prototype; and (c) Finished fabric design.

\section{Conclusion}

In this paper, endeavours were taken to substitute the traditional weaving process of the Chakma's design by semi-automated rapier loom. Digitising Chakma's pattern through JCAD oriented designing, and manufacturing system will enhance the aesthetic, feel and look of fabric. In this project, weaving of a single design on the fabric is possible by the rapier loom, but for complicated design, it is not possible to weave. Future work using jacquard machine can be done to weave a fabric containing multiple Chakma's designs. Introducing Chakma's fabrics can be digitised in more accurate ways than producing by conventional methods. Production of these extra-ordinary Chakma's clothes by automation mode would improve its aesthetics and provides with flexible end uses.

\section{Acknowledgements}

The project supported by the Department of Textile Engineering, Zhejiang Sci-Tech University, Hangzhou, China. We sincerely like to thank Professor Ding Xin Bo and Professor Xu for their support in supplying yarn, technical support for complete the project.

\section{Conflicts of Interest}

The authors declare no conflicts of interest regarding the publication of this paper.

\section{References}

[1] Chen, Q., Sun, H., Zhu, S., et al. (2017) Design of Dai's Patterned Yarn Jacquard Fabric. Textile Science and Technology Progress, No. 5, 28-31. (In Chinese)

[2] Zhang, X.Y. (2004) Reformation of the Prototype and Its Product Development. Sichuan Silk, No. 3, 14-15. (In Chinese)

[3] Jahirul, H. (2013) Cultural History of the Chakma Ethnic Tribe in Mizoram: A Study. International Journal of Computer Applications in Engineering Sciences, $\mathbf{3}$, 3. 
[4] Arshi, D. (2002) Woven Textile as Art: An Examination of the Revivel of Weaving in the Chittagong Hill Tracts. Concordia University, Canada, 6-13.

[5] Akter, A. (2019) Miscellaneous Fashion Trend of Indigenous. The Daily Jugantor, Bangladesh, 6.

[6] Hou, X.W. and Li, Y.T. (2010) On the Characteristics of the Indian Costumes by Sari. Progress in the Textile Science and Technology, No. 4, 82-84.

[7] Yang, T.T. (2013) Analysis of Traditional Women's Clothing in India. Shandong Textile Economy, No. 6, 73-74.

[8] Xu, H.M. (2011) Research on Image Recording in the Inheritance of National Costume Culture. Southwest University, Chongqing.

[9] Li, J. and Cai, Y.L. (2019) Analysis of African Traditional Costumes and Their Influences. Science and Technology, No. 3, 187.

[10] Zhang, W. (2004) Overview of Textile and Garment Industry in Bangladesh. Textile Information Weekly, No. 38, 39-40.

[11] Xu, S., Cheng, Y. and Gao, X. (2010) The Working Principle and Troubleshooting of Electronic Prototypes. Textile Industry and Technology, 39, 35-37.

[12] Li, Z. (1993) Effective Control of Yarn Tension. Foreign Textile Technology (Textiles), No. 11, 18-19.

[13] Jia, H.P. (2001) Realizing Tension Control in Yarn Process. Foreign Textile Technology, No. 12, 31-35.

[14] Yan, Y.F. (2010) Development of Warping Technology. Modern Silk Science and Technology, 25, 15-16+26.

[15] Guo, H.Y. (2012) Key Points of Warping Tension Control. Cotton Textile Technology, 40, 50-52.

[16] Liang, Q.J. (1997) Controlling the Weft Tension and Improving the Efficiency of the Loom. Jinan Textile Chemical Fiber Technology, No. 2, 43-48.

[17] Zhong, Y.R. (2009) Automatic Sample Machine Electric Lifting System. Shanghai Textile Technology, 37, 27-28+38. 